\title{
THE MORSE LEMMA FOR BANACH SPACES
}

\author{
BY RICHARD S. PALAIS ${ }^{1}$
}

Communicated April 28, 1969

Theorem. Let $V$ be a Banach space, $O$ a convex neighborhood of the origin in $V$ and $f: \theta \rightarrow R$ a $C^{k+2}$ function ( $k \geqq 1$ ) having the origin as a nondegenerate critical point, with $f(0)=0$. Then there is a neighborhood $U$ of the origin and $a C^{k}$ diffeomorphism $\phi: U \rightarrow 0$ with $\phi(0)=0$ and $(D \phi)_{0}=$ the identity map of $V$ such that for $x \in U f(\phi(x))=\frac{1}{2}\left(D^{2} f\right)_{0}(x, x)$.

REMARK. The above theorem is a classical result of Marston Morse in the case that $V$ is finite dimensional and was generalized by the author to the case that $V$ is a Hilbert space [1], [3]. The latter proof makes use of operator theory in Hilbert space and does not extend in any obvious way to more general Banach spaces. The proof we give below is completely elementary and works for arbitrary $V$ of course. Recent developments in the calculus of variations from a Banach manifold point of view (see for example [4]) make it desirable to have the theorem in this degree of generality.

The technique behind our proof was pioneered by J. Moser in a somewhat different and finite dimensional setting [2]. The present paper was inspired by a recent result of $A$. Weinstein [5] where Moser's method is adapted to the Banach manifold setting.

Put $f=f^{1}$ and define $f^{0}: \theta \rightarrow R$ by $f^{0}(x)=\frac{1}{2}\left(D^{2} f\right)_{0}(x, x)$. Define $f^{t}: \mathcal{O} \rightarrow R \quad 0 \leqq t \leqq 1$ by $f^{t}=f^{0}+t\left(f^{1}-f^{0}\right)$ and note that $(t, x) \mapsto f^{t}(x)$ is a $C^{k+2}$ map of $I \times \mathcal{O} \rightarrow R$. Note also that if we define $\dot{f}^{t}: \mathcal{O} \rightarrow \boldsymbol{R}$ by $\dot{f}^{t_{0}}(x)$ $=d /\left.d t\right|_{t=t_{0}} f^{t}(x)$ then clearly $\dot{f}^{t}=f^{1}-f^{0}$.

Moser's trick, suitably adapted, is to look for a smooth oneparameter family $\phi_{t}$ of $C^{k}$ local diffeomorphisms, defined in a neighborhood $U$ of 0 , with $\phi_{0}$ the identity, such that $f^{t} \circ \phi_{t}=f^{0}$ in $U$. This, of course, gives the theorem by taking $\phi=\phi_{1}$, since $f=f^{1}$. On its face we seem to have replaced our problem by a harder one; however, the nonlinear equation $f^{t} \circ \phi_{t}=f^{0}$ for $\phi_{t}$ is equivalent to $d / d t\left(f^{t} \circ \phi_{t}\right)=0$ which, as we shall see, is in turn equivalent to $D f^{t}\left(X^{t}\right)=-\dot{f}^{t}$, where $X^{t}$ is the time dependent vector field generating $\phi_{t}$. The latter is a linear equation for $X^{t}$ which can be solved explicitly.

Suppose then that $X^{t}, 0 \leqq t \leqq 1$ is a $C^{k}$ time dependent vector field on $\mathcal{O}$ (i.e. $(t, x) \mapsto X_{x}^{t}$ is a $C^{k}$ map of $I \times \mathcal{O} \rightarrow V$ ) with $X_{0}^{t}=0$ and $\left(D X^{t}\right)_{0}$ $=0$. Let $t \mapsto \phi_{t}(x)$ be the maximum integral curve of $X^{t}$ starting at

\footnotetext{
${ }^{1}$ Research supported in part by Air Force grant AFOSR 68-1403.
} 
$x \in \mathcal{O}$ (see [1, Chapter VI, §3]). Since $X_{0}^{t}=0$ it follows that $\phi_{t}(0)$ is defined and equals 0 for $0 \leqq t \leqq 1$, and since the set of $(t, x) \in I \times \mathcal{O}$ for which $\phi_{t}(x)$ is defined is open (see [1, p. 137]) there is a neighborhood $U$ of 0 such that $\phi_{t}(x)$ is defined for $(t, x) \in I \times U$; and by [1, p. 137] $(t, x) \mapsto \phi_{t}(x)$ is a $C^{k}$ map of $I \times U \rightarrow \mathcal{O}$. Since $\phi_{0}$ is the identity map of $U,\left(D \phi_{0}\right)_{0}$ is the identity map of $V$, and since $\left(D X^{t}\right)_{0}=0$ it follows from $\left[1\right.$, p. 135] that $\left(D \phi_{t}\right)_{0}=$ identity map of $V, 0 \leqq t \leqq 1$. If $g: \theta \rightarrow R$ is a $C^{1}$ map we note that by definition of $\phi_{t}$ we have

$$
\left.\frac{d}{d t}\right|_{t=t_{0}} g\left(\phi_{t}(x)\right)=X_{\phi_{t_{0}}(x)}^{t_{0}} g \text {. }
$$

Now suppose $(t, x) \mapsto g(t, x)=g^{t}(x)$ is a $C^{1}$ map of $I \times \mathcal{O} \rightarrow R$ and define $\dot{g}^{t}(x)=d(g(t, x)) / d t$ and $F(t)=g^{t} \circ \phi_{t}(x)=g\left(t, \phi_{t}(x)\right)$. Then by elementary calculus

$$
\begin{aligned}
F^{\prime}\left(t_{0}\right) & =\left.\frac{d}{d t}\right|_{t=t_{0}} g\left(t, \phi_{t_{0}}(x)\right)+\left.\frac{d}{d t}\right|_{t=t} g\left(t_{0}, \phi_{t}(x)\right) \\
& =\dot{g}^{t_{0}}\left(\phi_{t_{0}}(x)\right)+X_{\phi_{\theta_{0}}(x)}^{t_{9}} g^{t_{0}} .
\end{aligned}
$$

This proves

Lemma 1. If $(t, x) \mapsto g^{t}(x)$ is a $C^{1}$ map of $I \times \mathcal{O} \rightarrow R$ then for $(t, x)$ $\in I \times U$

$$
\frac{d}{d t}\left(g^{t} \circ \phi_{t}(x)\right)=\left(\dot{g}^{t}+X^{t} g^{t}\right) \circ \phi_{t}(x) .
$$

In particular if $X^{t} g^{t}=-\dot{g}^{t}, 0 \leqq t \leqq 1$ then $g^{t} \circ \phi_{t}(x)=g^{0}(x),(t, x)$ $\in I \times U$.

Let $L\left(V, V^{*}\right)$ denote the Banach space of continuous linear maps of $V$ into $V^{*}, L_{s}\left(V, V^{*}\right)$ the closed subspace of those $S$ such that $\langle u, S v\rangle=\langle v, S u\rangle$ for all $u, v \in V$ (where $\langle$,$\rangle is the natural pairing of$ $\left.V \times V^{*} \rightarrow R\right)$, and Iso $\left(V, V^{*}\right)$ the open subset of $L\left(V, V^{*}\right)$ consisting of $S$ mapping $V$ isomorphically onto $V^{*}$. We recall that $S \rightarrow S^{-1}$ is a $C^{\infty}$ diffeomorphism of Iso $\left(V, V^{*}\right)$ onto Iso $\left(V^{*}, V\right)$ since it is given locally by a convergent power series.

Under the natural identification of the Banach space $L^{2}(V, R)$ of continuous bilinear functionals on $V$ with $L\left(V, V^{*}\right)$ (defined by $B \mapsto S_{B}$, where $\left.B(u, v)=\left\langle u, S_{B} v\right\rangle\right) L^{2}(V, R)$, the closed subspace of symmetric bilinear functionals is mapped isomorphically onto $L_{s}\left(V, V^{*}\right)$. Now $D^{2} f: \theta \rightarrow L_{s}^{2}(V ; R)$ is a $C^{k}$ map, so composing with the above identification we have a $C^{k}$ map $S: \mathcal{O} \rightarrow L_{s}\left(V, V^{*}\right)$ defined by the condition $D^{2} f_{x}(u, v)=\left\langle u, S_{x} v\right\rangle=\left\langle v, S_{x} u\right\rangle$. By definition of a non- 
degenerate critical point $S_{0} \in$ Iso $\left(V, V^{*}\right)$. We define a $C^{k}$ map $S^{1}: \mathcal{O} \rightarrow L_{s}\left(V, V^{*}\right)$ by $S_{x}^{1}=\int_{0}^{1} S_{t x} d t$, and a $C^{k}$-map $(t, x) \mapsto S_{x}^{t}$ of $I \times \mathcal{O}$ $\rightarrow L_{s}\left(V, V^{*}\right)$ by $S_{x}^{t}=S_{0}+t\left(S_{x}^{1}-S_{0}\right)$. Since $S_{0}^{t}=S_{0} \in$ Iso $\left(V, V^{*}\right)$ and Iso $\left(V, V^{*}\right)$ is open in $L\left(V, V^{*}\right)$ it follows that, replacing $\theta$ if necessary by a smaller convex neighborhood of 0 , we can assume that $S_{x}^{t}$ $\in$ Iso $\left(V, V^{*}\right)$ for all $(t, x) \in I \times \mathcal{O}$. Since $S \mapsto S^{-1}$ is a $C^{\infty}$ map of Iso $\left(V, V^{*}\right) \rightarrow$ Iso $\left(V^{*}, V\right)$ it follows that if we define $T_{x}^{t} \in L(V, V)$ by $T_{x}^{t}=\left(S_{x}^{t}\right)^{-1} \int_{0}^{1}\left(S_{s x}^{0}-S_{s x}^{1}\right) s d s$ then $(t, x) \mapsto T_{x}^{t}$ is a $C^{k}$ map of $I \times \mathcal{O}$ into $L(V, V)$ and clearly $T_{0}^{t}=0$. Finally, we define $X_{x}^{t} \in V$ by $X_{x}^{t}=T_{x}^{t} x$, and it follows that $(t, x) \mapsto X_{x}^{t}$ is a $C^{k}$ map of $I \times \mathcal{Q} \rightarrow V$, that $X_{x}^{t}=0$ and that $\left(D X^{t}\right)_{0}=0$. Let $(t, x) \mapsto \phi_{t}(x)$ be the $C^{k}$ map $I \times U \mapsto \mathcal{O}$ defined as above by integrating $X^{t}$. According to Lemma 1 , if we show that $X^{t} f^{t}=-\dot{f}^{t}=f^{0}-f^{1}$ then it will follow that in $U f^{t} \circ \phi_{t}=f^{0}$ and in particular $f \circ \phi=f^{0}$ where $\phi=\phi_{1}$, which will prove the theorem. Equivalently it remains to show that for all $(t, x) \in I \times \mathcal{O} D f_{x}^{t}\left(X_{x}^{t}\right)$ $=f^{0}(x)-f^{1}(x)$.

Lemma 2. If $x \in \mathcal{O}$ and $v \in V$ then

$$
D f_{x}^{t}(v)=\left\langle x, S_{x}^{t} v\right\rangle \quad 0 \leqq t \leqq 1 .
$$

Proof. Since 0 is a critical point of $f=f^{1} D f_{0}^{1}=0$ and so

$$
\begin{aligned}
D f_{x}^{1}(v) & =\int_{0}^{1} \frac{d}{d s} D f_{s x}^{1}(v) d s=\int_{0}^{1} D^{2} f_{s x}^{1}(x, v) d s=\int_{0}^{1}\left\langle x, S_{s x} v\right\rangle d s \\
& =\left\langle x, S_{x}^{1} v\right\rangle .
\end{aligned}
$$

On the other hand clearly $D f_{x}^{0}(v)=\left\langle x, S_{0} v\right\rangle$. Since $D$ is linear and $f^{t}=f^{0}+t\left(f^{1}-f^{0}\right)$ while $S_{x}^{t}=S_{0}+t\left(S_{x}^{1}-S_{0}\right)$ the lemma follows. q.e.d.

We can now complete the proof of the theorem. Recalling that by definition $X_{x}^{t}=T_{x}^{t} x$ where $T_{x}^{t}=\left(S_{x}^{t}\right)^{-1} \int_{0}^{1}\left(S_{s x}^{0}-S_{s x}^{t}\right) s d s$ it follows from Lemma 2 that

$$
D f_{x}^{t}\left(X_{x}^{t}\right)=\int_{0}^{1}\left\langle x,\left(S_{s x}^{0}-S_{s x}^{1}\right) x\right\rangle s d s=\int_{0}^{1}\left(\left\langle s x, S_{s x}^{0} x\right\rangle-\left\langle s x, S_{s x}^{1} x\right\rangle\right) d s
$$

so applying Lemma 2 a second time we have

$$
\begin{aligned}
D f_{x}^{t}\left(X_{x}^{t}\right) & =\int_{0}^{1} D\left(f^{0}-f^{1}\right)_{s x}(x) d s=\int_{0}^{1} \frac{d}{d s}\left(\left(f^{0}-f^{1}\right)(s x)\right) d s \\
& =f^{0}(x)-f^{1}(x),
\end{aligned}
$$

since $f^{0}(0)=f^{1}(0)=0$. 


\section{REFERENCES}

1. S. Lang, Analysis. II, Addison-Wesley, Reading, Mass., 1968.

2. J. Moser, On the volume elements on a manifold, Trans. Amer. Math. Soc. 120 (1965), 286-294.

3. R. Palais, Morse theory on Hilbert manifolds, Topology 2 (1963), 299-340.

4. - Foundations of global non-linear analysis, Benjamin, New York, 1968.

5. A. Weinstein, Symplectic structures on Banach manifolds, Bull. Amer. Math Soc. (to appear).

Brandeis University, Waltham, Massachusetts 02154 aNd

Institute for Advanced Study, Princeton, New Jersey 08540 